\title{
IMPEDANCE SPECTROSCOPY OF PIEZOELECTRIC ACTUATORS
}

\author{
C.R. Bowen, M. Lopez-Prieto, S. Mahon* and F. Lowrie* \\ Faculty of Engineering and Design, Department of Materials Science and Engineering, University of \\ Bath, Bath, Somerset BA2 7AY, UK *Mechanical Science Sector, DERA Farnborough, \\ Hampshire GU14 OLX, UK
}

(Received October 26, 1999)

(Accepted in revised form December 13, 1999)

Keywords: Multilayers; Piezoelectricity; Non-destructive testing; Ceramic; Functional

\section{$\underline{\text { Introduction }}$}

Multi-layer actuators are used for applications which require large displacements at comparatively low drive voltages (50-200V) and low power consumption compared to monolithic materials. It has been reported in the literature that delamination of the electrode layers during processing can act as nuclei for crack propagation. Mechanisms of processing induced defects are binder burnout, green state delamination due to inadequate adhesion of the electrode and piezoelectric layer, catalytic reactions of electrode metals with organic additives during burnout and sintering shrinkage mismatch (1). In addition to processing induced defects, the composite structure of these devices and the electrode configuration can result in the actuators suffering from fatigue and degradation of performance as a function of time (2-5). The fatigue of multi-layer actuators is thought to be due to the development of internal stresses around the inactive and active regions of the piezoelectric material at the electrode edges $(6,7)$. In addition to multi-layers, internal stresses and processing defects are developed in other piezoelectric actuators such as bimorphs, moonies and piezocomposites. Therefore, there is a need to non-destructively detect defects in piezoelectric actuators during production and to detect the development of fatigue damage during service (self-validation) in a simple and rapid manner.

Non-destructive testing methods to assess the reliability of multi-layer actuators have included direct measurement of insulation resistance (IR), the measurements of induced displacement and acoustic emission, all of which have been used with some success to monitor damage and degradation of multi-layer actuators $(2,4)$. A promising technique is impedance spectroscopy (IS), ie. the measurement of electrical impedance as a function of frequency. A multi-layer actuator will undergo resonance which is determined by the nature of the materials and the dimensions of the device. It is expected that a change in the resonance characteristics of the actuators will be observed as the degree of damage increases in the device $(8-11)$. The aim of this paper is to examine the change in resonance behaviour of typical multi-layer actuators due to the presence of delaminations and/or cracks introduced into the device. Experimental observations of impedance response will be compared with direct observations of defects using optical and scanning electron microscopy of sectioned devices. Various methods used to present impedance data such as 'Bode' diagrams or 'complex plane' (Nyquist plots) will be examined to determine which is most applicable in examining any change in resonance characteristics of a multi-layer actuator due to damage. 


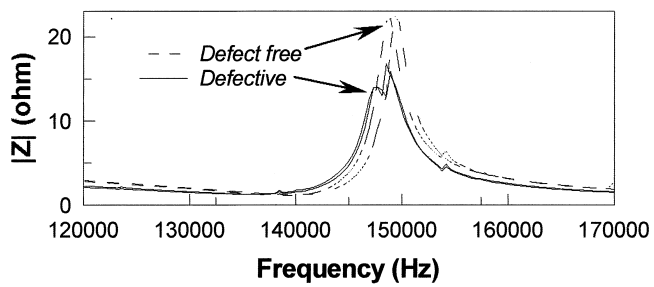

(a)

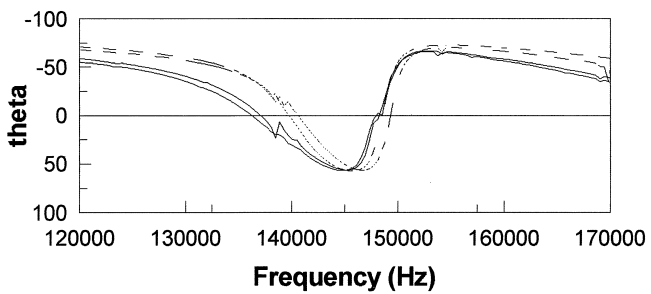

(b)

Figure 1. Bode representation of (a) modulus of impedance $|\mathrm{Z}|$ and (b) phase angle $(\theta)$ as a function of frequency. Notice that the defective multi layer actuators have a lower maximum $|\mathrm{Z}|$ at anti-resonance $(\sim 150 \mathrm{kHz})$.

\section{Experimental}

A range of multi-layer actuators of different "quality" were deliberately fabricated and supplied by a commercial manufacturer. The actuators in this research had a cross section of $10 \mathrm{~mm} \times 10 \mathrm{~mm}$ and a thickness of $2 \mathrm{~mm}$, consisting of 30 electrodes with an electrode spacing of approximately $85 \mu \mathrm{m}$ through the thickness. The actuators were fabricated from a soft piezoelectric material with high strain response.

Impedance spectroscopy was undertaken using a Solartron 1260 Frequency Response Analyser and 1296 Dielectric Interface. A sinusoidal AC signal of $0.1 \mathrm{~V}$ was applied to the device in a frequency range above and below the resonant frequency of the device. A small signal is required to prevent depoling of the device during testing. The resonant frequency was determined from a calculation based on the dimensions of the actuator (assuming width resonance) and an initial large range frequency sweep.

To validate that impedance measurements were accurately detecting defects within the actuators, destructive sectioning and final device inspection was undertaken using a diamond cutting wheel, polishing the sectioned surface to $1 \mu \mathrm{m}$ with diamond paste and subsequent examination using optical microscopy and Scanning Electron Microscopy (SEM) using a JEOL T330. Damage was also introduced deliberately into some of the devices using a Vickers hardness indentation at an applied load of $30 \mathrm{~kg}$.

\section{Results and Discussion}

Figure 1a shows the modulus of impedance $|\mathrm{Z}|$ and loss angle $(\theta)$ as a function of frequency for four different multi-layer actuators. The experimentally observed resonance frequencies of $148 \mathrm{kHz}$ agree well with calculated values of $140 \mathrm{kHz}$ based on the equation,

$$
f=\frac{1}{\sqrt{4 \cdot \rho \cdot t^{2} \cdot s_{11}^{E}}}
$$


TABLE 1

Maximum Modulus of Impedance Values of Multi-Layer Actuators Tested

\begin{tabular}{lcc}
\hline \multicolumn{1}{c}{ Sample } & $\begin{array}{c}\text { Maximum impedance } \\
(\text { ohm })\end{array}$ & $\begin{array}{c}\text { Frequency } \\
(\mathrm{kHz})\end{array}$ \\
\hline Defect Free 1 & 21.81 & 148.5 \\
Defect Free 2 & 22.41 & 149.5 \\
Defect 1 & 16.74 & 148.5 \\
Defect 2 & 15.84 & 148.9 \\
\hline
\end{tabular}

where,

$\rho=$ density of piezoelectric material $\left(7500 \mathrm{~kg} \mathrm{~m}^{-3}\right)$

$\mathrm{t}=$ multi-layer width $(10 \mathrm{~mm})$

$\mathrm{s}_{11}^{\mathrm{E}}=$ compliance of piezoelectric material at constant electric field $\left(16.4 \times 10^{-12} \mathrm{~Pa}\right)$

$\mathrm{f}=$ width resonance frequency $(\mathrm{Hz})$

For two of the actuators tested there is a sharp resonant behaviour with comparatively high values of maximum impedance at the parallel resonance frequency, as indicated in Figure 1a. For the two remaining actuators the resonance tests results in lower maximum values of impedance at resonance. The results are summarised in Table 1. Based on the assumption that defects such as cracks or delaminations will damp the resonance behaviour of the actuators (rather than changing the actual resonant frequency), the actuators with the sharp resonance and highest maximum impedance will be labelled "defect free," while the actuators with the damped resonance will be assumed to have a "defect." Figure $1 \mathrm{~b}$ shows that there is also an observed, but less significant, difference in the change of loss angle $(\theta)$ as a function of frequency as the "defect free" and "defect" containing actuators pass through resonance.

The differences observed between the "defect free" and "defective" actuators in $|\mathrm{Z}|$ and $\theta$ as a function of frequency can be combined by plotting a complex impedance plot of $Z^{\prime}$ versus $Z^{\prime \prime}$ where $Z^{\prime}=|Z| \cos \theta$, the real part if the impedance and $Z^{\prime \prime}=|Z| \sin \theta$, the imaginary part of the impedance. A complex impedance plot is shown in Figure 2 in which a complete circle is drawn and the device passes through resonance. The intercept of the circle with the $\mathrm{Z}^{\prime}$ axis is the equivalent of the maximum of $|\mathrm{Z}|$ in the impedance plots in Figure 1a. The intercept with $\mathrm{Z}^{\prime}$ is the point where the impedance is in phase with applied signal and the loss angle is zero (compare intercepts in Figure 2 with maximum impedance values in Table 1). Therefore, actuators with defects or damage exhibit damped resonance and describe smaller diameter complex impedance plots, as seen in Figure 2. The advantage of the complex impedance representation of impedance data is that small changes in behaviour can be readily observed and detected, compared to the Bode presentation (i.e. $|\mathrm{Z}|$ and $\theta$ versus frequency). The complex impedance representation is also an advantage when testing and comparing large numbers of specimens, for instance during device production.

The samples were subsequently sectioned and examined under an SEM to validate whether impedance spectroscopy was accurately detecting defects in the actuators. Figure 3 shows SEM images of the actuators labelled "Defect 1" and "Defect 2." Figure 3a clearly shows a crack, which is propagating along the ceramic between the metallic electrodes in the sample "Defect 1." In this case the observed defect is of the order of $1 \mathrm{~mm}$. Figure $3 \mathrm{~b}$ shows that the sample "Defect 2" has a missing electrode layer. This would produce an area, which does not experience an electric field, as the electrodes above and below, would be of the same polarity. There would therefore be a significant "dead zone" within the actuator. No defects were observed in the "defect free" actuators with a sharp resonance and higher values of maximum impedance. 


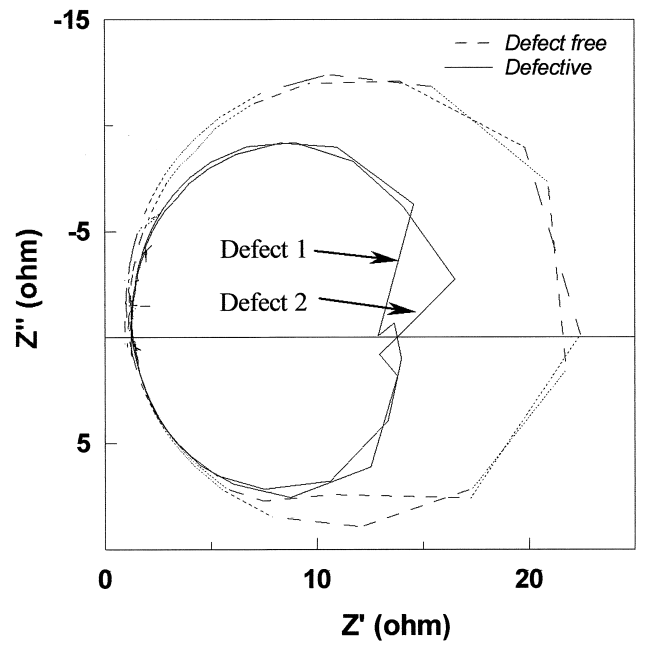

Figure 2. Complex impedance spectrum of the same data represented in Figure 1 where $Z^{\prime}=|Z| \sin \theta$ and $Z^{\prime \prime}=|Z| \cos \theta$. The defect free actuators are characterised by large impedance loops corresponding to a larger maximum impedance at anti resonance.

Impedance spectroscopy seems to have been successful in rapidly and non-destructively detecting defects within multi-layer actuators. To further validate these observations, damage was deliberately and systematically introduced into an actuator and the subsequent change in resonance measured. Figure 4 shows the change in the complex impedance plot as $30 \mathrm{~kg}$ Vickers indents are introduced into the device. The diameter of the complex impedance plot is reduced, and the degree of damping increases as damage is introduced into the device. Impedance spectroscopy measurements have also been compared with ultrasonic C scan imaging on these actuators, with good agreement (12).

\section{Conclusions}

It has been observed that examination of the impedance spectrum of multi-layer actuators in the width resonance has been able to rapidly and non-destructively detect defects within the devices. The test has been used to detect defects induced by processing (such as poling cracks) and deliberately introduced defects by indentation. The impedance results have been verified by direct observations of defects by microscopy.

Impedance spectroscopy detects damage by using the damping nature of the defect and the resultant change in the resonance behaviour of the device. The examination of impedance-frequency data in the form of a complex impedance plot ( $Z^{\prime}$ versus $\left.Z^{\prime \prime}\right)$ has shown to be the most useful in rapidly determining small changes in resonant behaviour. Complex impedance curves present both $|Z|$ and $\tan \theta$ data as a function of frequency and damaged actuators are clearly detected by the small diameter circle which they produce.

If the resonance frequency of the multi layer actuator is known (from equation 1), the impedance spectrum and the 'health' of the actuator can be determined in short measurement times. Rapid test times are of importance when the actuators are produced in large quantities. The test could be developed to detect delaminations and other defects during production and/or the monitoring of fatigue during in-service operation, providing that any clamping of the device does not prevent the resonance from occurring. The method could also be suited to other piezoelectric devices such and benders or composites. Further work will evaluate the sensitivity of the technique (as the defects detected here are 


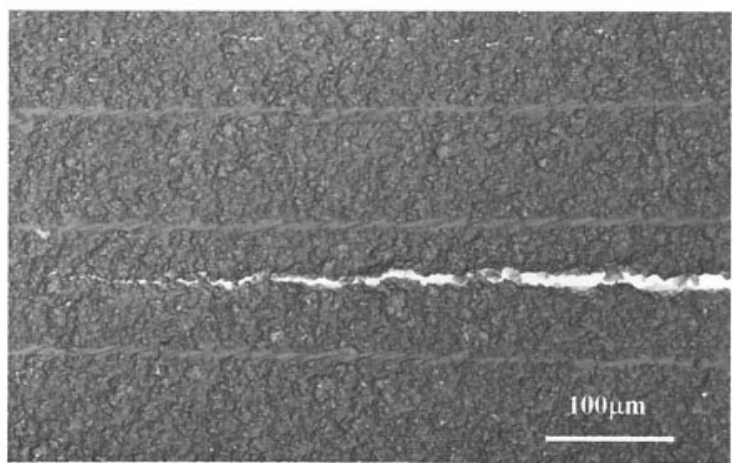

(a)

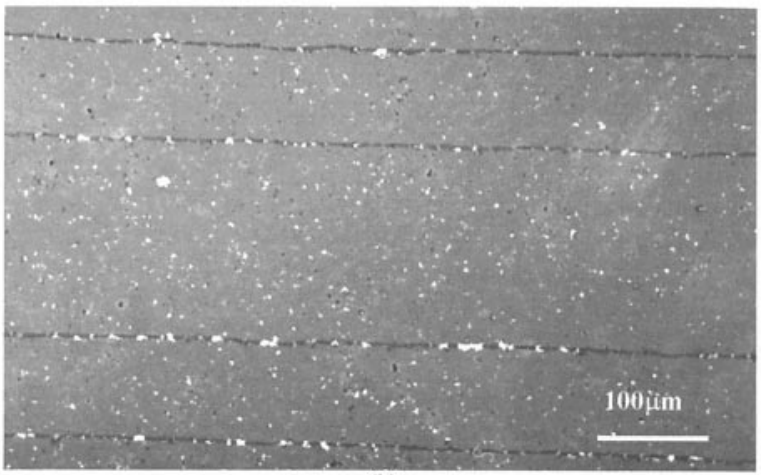

(b)

Figure 3. (a) SEM of multi layer actuator "Defect 1" showing a crack propagating within the ceramic layer. (b) SEM image of a missing electrode layer within the multi layer actuator "Defect 2", which leads to a damped impedance spectrum compared to defect free actuators. The corresponding impedance spectra are shown in Figures 1 and 2.

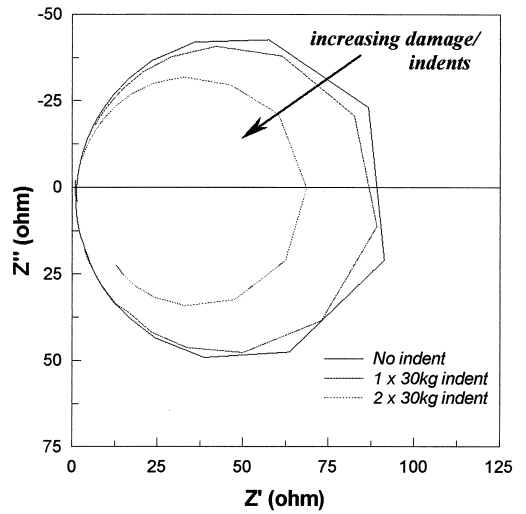

Figure 4. Complex impedance plots shown the effect of defects introduced into a multi layer by indentation. Diameter and intercept with $\mathrm{Z}^{\prime}$ axis decreases with increasing damage. 
greater than $100 \mu \mathrm{m})$, the types of defect detectable by impedance spectroscopy and whether defects introduce non-linear behaviour (13) into the impedance response (a change in complex impedance as a function of applied voltage).

\section{Acknowledgments}

This work was carried out as part of Technical Group 04 of the MOD Corporate Research Programmes.

\section{References}

1. J. G. Peppin, W. Borland, P. O’Callaghan, and R. J. S. Young, J. Am. Ceram. Soc. 72, 2287 (1989).

2. H. Aburatani, S. Harada, K. Uchino, A. Furuta, and Y. Fuda, Jpn. J. Appl. Phys. 33, 3091 (1994).

3. A. Furuta and K. Uchino, J. Am. Ceram. Soc. 76, 1615 (1993).

4. H. Aburatani, S. Yoshikawa, K. Uchino, and J. W. C. de Vries, Jpn. J. Appl. Phys. 37, 204 (1998).

5. K. Uchino and S. Takahashi, Curr. Opin. Sol. State Mater. Sci. 1, 698 (1996).

6. S. Takahashi, A. Ochiai, M. Yonezawa, T. Yano, T. Hamastuki, and I. Fukui, Ferroelectrics. 50,507 (1983).

7. X. Gong and Z. Suo, J. Mech. Phys. Sol. 44, 51 (1996).

8. O. Boser, P. Kellawon, and R. Geyer, J. Am. Ceram. Soc. 72, 2282 (1989).

9. O. Boser, Adv. Ceram. Mater. 2, 167 (1987).

10. B. Zickgraf, G. A. Schneider, and F. Aldinger, in ISAF '94-Proceedings of the 9th IEEE International Symposium on Application of Ferroelectrics, 325 (1994).

11. B. Zickgraf, Ermüdungsverhalten von Multilayer-Aktoren aus Piezokeramik, Fortschrtt-Berichte VDI, Reihe 18, Nr. 191, VDI Verlag, Düsseldorf (1996).

12. C. R. Bowen, M. Lopez Prieto, and S. W. Mahon, Ferroelectrics. accepted.

13. R. A.Guyer and P. A. Johnson, Phys. Today. 52, 30 (1999). 